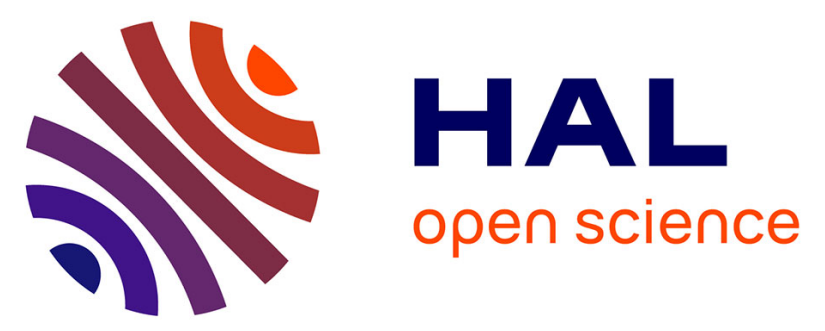

\title{
Industry 4.0: Educational platforms disseminations in South-East Asia in the field of Automation
}

Jean-Marc Thiriet, Denis Genon-Catalot, Stéphane Mocanu, Hamed Yahoui

\section{To cite this version:}

Jean-Marc Thiriet, Denis Genon-Catalot, Stéphane Mocanu, Hamed Yahoui. Industry 4.0: Educational platforms disseminations in South-East Asia in the field of Automation. EAEEIE 2021 30th Annual Conference of the European Association for Education in Electrical and Information Engineering (EAEEIE), Sep 2021, Prague, Czech Republic. 10.1109/EAEEIE50507.2021.9530861. hal-03429657

\section{HAL Id: hal-03429657 https://hal.science/hal-03429657}

Submitted on 15 Nov 2021

HAL is a multi-disciplinary open access archive for the deposit and dissemination of scientific research documents, whether they are published or not. The documents may come from teaching and research institutions in France or abroad, or from public or private research centers.
L'archive ouverte pluridisciplinaire HAL, est destinée au dépôt et à la diffusion de documents scientifiques de niveau recherche, publiés ou non, émanant des établissements d'enseignement et de recherche français ou étrangers, des laboratoires publics ou privés. 


\section{Industry 4.0: Educational platforms disseminations in South-East Asia in the field of Automation}

\author{
Jean-Marc Thiriet \\ Gipsa-lab \\ Univ. Grenoble Alpes \\ Grenoble, France \\ jean-marc.thiriet@univ-grenoble- \\ alpes.fr \\ Hamed Yahoui \\ Univ. Claude Bernard Lyon 1 \\ Lyon, France \\ hamed.yahoui@univ-lyon1.fr
}

\author{
Denis Genon-Catalot \\ LCIS \\ Univ. Grenoble Alpes \\ Valence, France \\ denis.genon-catalot@univ-grenoble- \\ alpes.fr
}

\author{
Stéphane Mocanu \\ $L I G, E N S E 3$, \\ Univ. Grenoble Alpes \\ Grenoble, France \\ stephane.mocanu@grenoble-inp.fr
}

\begin{abstract}
Asean-Factori 4.0 is an ERASMUS+ Key Action 2 project managed by Université Claude Bernard Lyon 1 and regrouping European partners in Lyon, Ruse (Bulgaria) and Grenoble together with South-East Asian partners in Cambodia, Laos and Thailand. The purpose is a collaboration between the European and Asian partners in order to enrich Asian curricula and transfer up-to-date platform in the field of automation for teaching the most recent engineering concepts such as Factory 4.0 or Cyber-security of cyber-physical systems. Various actions have been held and are on the way within this project.
\end{abstract}

Keywords- automation, industry 4.0, cyber-security, Asean countries, key action 2 project

\section{INTRODUCTION}

The "Asean-Factori 4.0" projects is an European project accepted initially for 3 years from 2020 to end 2022 by the European Commission under the framework of the ERASMUS+ Key Action 2 CBHE (Capacity Building in High Education) program. Due to the pandemic situation which has stopped any travel, the project end will be delayed. This project aims at enhancing existing curricula in the field of automation in the 6 Asian (South-East Asian) partners of the project coming from three countries: Cambodia, Laos and Thailand. In these universities, some curricula exist in the field of automation, but there are no platforms, needed to train students, related to that. Our project consists in defining pertinent platforms, together with our Asian partners, to propose or adapt pedagogical resources (courses, labs textbooks) to accompany the deployment of these platforms in their universities and to train trainers. Most of the money available in the project is dedicated to buy platforms for Asian partners. It will be a way for them to be at the state of the art in the field of automation, Industry 4.0, cyber-security of cyber-physical systems, with updated courses and related actual platforms to train students.

In order to achieve these purposes and to target the end users, several actions are deployed. We can identify four end users within this project. The final end users are the companies, employers and employees, in order to participate to the socio-economic development of the related countries and economies. To reach employers, some training actions, specifically dedicated to them, using the platforms which will be deployed, will be organized during the last year of the project. The second target users are students who will become employees (also sometimes employers). The students are targeted at two levels within the project: bachelor students and master students. For bachelor students, the European teams of the project (Ruse university in Bulgaria, and Univ. Grenoble Alpes in France) have prepared seminars, in order to raise their awareness of the field of Industry 4.0 ; for master students, the activities will concern "projects" which will be managed by a bi-national (Asia-Europe) team of teachers, around the platforms developed within the project; the practical teaching or Project-Based Learning is important at this stage and is one main purpose of the project: technology transfer, competence transfer. In order to ensure the sustainability of the actions carried out after the end of the project, training of trainers is an important action to be held during the second year of the project. Trainers are the third target of the project, their training is a guaranty for the sustainability of the project beyond its end. The last end user is more oriented to the university decision-makers, with some actions in the assessment dimension.

The next section presents the organization. $3^{\text {rd }}$ section explains the seminar action. $4^{\text {th }}$ section focuses on the benchmark/platform transfer action. The fifth section discusses the involvement of students. Then the following section proposes new actions for the near future, and the paper ends with a conclusion.

\section{ORganization OF THE PROJECT}

The project duration is three years, we have delays due to the present pandemic and so the calendar has been reorganized. This year is dedicated to define the needed hardware, PLCs, input-output devices, specific processes. A training period will be organized in France for targeted Asian partners, probably in Fall 2022. This period will aim the future Asian teachers/trainers to learn and be able to use their future platforms. As it was mentioned before, the transferability of the platform and the appropriation by the Asian partners is the most important aspect of the project: for that, the components will be bought during this 2021 year.

So the platform components will be available in Asia at last at the summer 2022, which means that academic year 2022-2023 will be used by the Asian partners to install and begin to use the platform with students, under the remote supervision of the European partners. Before, in spring 2022, European partners will attend dedicated training seminars aiming at validating the work done by students and teachers 
on the platforms, bringing extra-expertise to Asian partners, and thinking to the organization of a Center of Excellence which is envisaged as a network of the Asian institutions in the future, the 6 institutions will have actually complementary different platforms.

The last year (2022-2023) will be also used for the validation of the deployment of the enhanced courses and new platforms in the Asian partners, and the setting of the Center of Excellence, also with intensive programs organized towards industrial partners.

\section{SEMINAR Actions TARgetting BACHELOR StUdENTS}

One of the activities of the project aims at raising bachelor student's awareness of the field of Industry 4.0. For that, a set of 6 seminars have been prepared by the European team. Ruse (Bulgaria) prepared 3 seminars [1] and Grenoble 3 seminars. The contents of these seminars deal globally around the concepts of Industry 4.0. We present in the next the three seminars proposed by Grenoble.

The first Grenoble seminar is entitled "Concept of Industry 4.0: some challenges and means". The first part recalls the story of Industry, back from Industry 1.0 to Industry 4.0: development of mechanization, then arrival of electricity and mass production, development of robotics from the $70 \mathrm{~s}$, finally wide deployment of communication systems. The challenges of standards in the field are also emphasised. An explanation of what is a PLC (Programmable Logic Controller) and its mains differences (industrial environment, input-output interfaces, specific network interfaces and specific firmware) with a classical computer are then presented. A presentation about the main cybersecurity challenges, taking account of the industrial system environment specificities, is then taught with a comparison with classical IT (Information Technology) environments and also rudiments of risk analysis and management are then provided. The next steps deal with various aspects of the functionalities which should be managed within the frame of Industry 4.0: not only production, but also maintenance, logistics, supervision. Some scientific concepts as well as a state of the art in the application field are given on these aspects. A focus is then given on the use of robots and cobots in the Industry 4.0 environment.

The second Grenoble seminar is entitled "Concept of Industry 4.0: PLC network extensions and real time Networks". This seminar is organized in 5 parts. In the first part and as an example, a real process is proposed, which is also an "environmental" process, a small Hydro Power Plant. On this type of process, water management is one of the main purposes. Various aspects such as instrumentation, control (for which PLCs are relevant tools), communication (especially wireless networks and distributed sensors considered as IoT nodes) are related to that. The second part deals with automation architectures. For that, first are explained the differences between a classical and an intelligent distributed architecture, with the related communication challenges. The communication is then developed. The various possible existing physical layers are presented. The OSI model is then explained, and the available protocols for the various layers. The fieldbus networks, widespread in the field of automation, are introduced, within the frame of the IEC 61158 standard. A focus is then given on Profibus, and finally the convergence between fieldbus networks and TCP/IP networks: Profinet, Industrial Ethernet,
Ethernet Powerlink, are explained. IEC 61850, which is a TCP/IP extension dedicated to electricity production and distribution is finally discussed. In the last part of this seminar, wireless networks are presented, together with LoRa. Finally, a general conclusion is given regarding the various networks which may exist in the Industry 4.0 context: industrial networks, wireless networks, classical TCP/IP networks together with a real-time extension...

The third slot of classes deals with cyber-security in the context of industrial systems. Cyber-security deals within two different contexts: the contexts of IT (Information Technology) in which the security is seen from a perspective of infrastructure or from a perspective of data, and OT (Operational Technology) in which the security is seen from the perspective of the operational aspect of the system. In the field of OT, with application in the field of Industrial Systems or Internet of Things, the consequence of an attack may be in the physical world, resulting possibly in an accident and catastrophe in the real world. The priorities regarding the cyber-security may so be different. The class then presents the concept of Information System and the various assets. The concept of attacks and vulnerabilities are then developed. Then some considerations are given in the field of energy, with some historical aspects and also specific issues. Some attack strategies are then explained: the 2003 Black-out, the 2010 Stuxnet, the Industroyer/Crashoverride. Then a systemic approach is proposed, based on the SCADA cyber-security awareness, taking account of the control, communication and security. Based on the 27000 standards, some strategies are then studied. The CIA (Confidentiality, Integrity, Availability) concept is used. Several security concepts (audit) are then presented. Cryptography is then presented: both the symmetric and asymmetric encryption mechanisms and their use to protect confidentiality and achieve signatures. Then a risk assessment methodology is proposed, in the field of cyber-security. The presentation ends with presentation of cyber-security tools such as Intrusion Detection Systems and Off-line security tools, and their use in the context of cybersecurity of cyber-physical systems.

The purpose of these seminars was to give the bachelor students an overview of the Industry 4.0 context, the functionalities of Industry 4.0, the need for an information system and a communication infrastructure, the possible network protocols used, and the cyber-security challenges in a cyber-physical environment.

\section{TRANSFERABILITY OF AUTOMATION PLATFORMS}

At Univ. Grenoble Alpes, we have a platform [2] [3] in the field which was presented in previous papers. The transfer of pedagogical platforms is an important aspect of the Asean Factori 4.0 project.

One of the purposes of the project is to set some Centers of Excellence in South-East Asia in the field of Industry 4.0, and to build a network of centers of excellence. The six Asian partners in the field of Automation will, each of them, receive a platform, co-designed in an Asian-European partnership, two of these platforms are designed by the Ruse partner in Bulgaria [1], one is designed by Lyon and three by Grenoble. In order to fit well with the needs of the Asian partners and give a meaning to the network of centers of excellence, it has been accepted that the platforms should illustrate various applications whereas the brands should be different from one center of excellence to another, in order for the network of 
centers of excellence to have a global knowledge and knowhow on the various technologies existing on the market. In Grenoble, the three selected platforms are described in the following parts.

The lab number 1 is a lab dedicated to the cyber-security of industrial systems. This lab is based on Schneider ${ }^{\circledR}$ technology. It is composed of a PLC, with direct InputOutputs and a CAN network interface. A distant HMI (Human Machine Interface) is used to illustrate an actual industrial situation and also to study and analyze the communication aspects: type of protocols used, ways the data are sent, show the possible use of cryptography... In order to test some cyber-security issues (vulnerabilities, attacks, protection), a Stormshield ${ }^{\circledR}$ firewall, which allows to take account industrial protocols and higher layer application aspects, is used in this lab. Various strategies, such as the use of a Demilitarized Zone (DMZ) can be configured and tested. An engine is part of the platform. It is actually possible to attack the engine, thanks to an integrity violation attack, and show technically the consequences in the physical world of an attack achieved in the digital world. This platform allows also achieving other applications such as the control of the engine, the remote supervision of the system thanks to the HMI, and other activities around automation.

The second lab is based on a Siemens ${ }^{\circledR}$ PLC. The main purpose of this platform is to study communication. It is composed of a PLC with inputs-outputs and a Profibus interface. An HMI is provided to allow remote supervision, and also specifically to study the network infrastructure and message exchanges. A network architecture is provided around a switch allowing to analyze all the messages exchanged by all the participants of the network (PLCs, HMIs, computers...), a firewall is also provided. One of the innovations of this lab is the use of a specific emulation process card. This consists in an embedded Linux system, together with a network interface controller (NIC), various digital and analog inputs-outputs, some leds and switches. The purpose of this emulation card is to be programmed, in order to run a simulated system. The NIC allows the emulation process card to dialog with the other devices of the installation, as a normal process. The input-output interfaces allow a direct physical connection between the PLC and the emulation process card, this is one of the most interesting aspects of this card, since from the PLC perspective, there is an actual connection with IO. The switches on the emulation process card allows to manually control the IO and leds are $\mathrm{ON}$ when their relative signal is $\mathrm{ON}$. A software is used on a PC to remote control the emulation process card. Thanks to this architecture, it is possible to test various types of processes, for instance in partnership with companies, without having to install the complete physical process; it gives the platform an interesting generalization.

The third platform is oriented towards wireless networks, with an application particularly in the field of smart buildings. This platform is based on a Wago ${ }^{\circledR}$ PLC. As for the other platforms, some Inputs-Outputs will be directly and physically connected to the PLC. Some wireless interfaces are provided, based on the EnOcean network. A Human-Machine Interface is provided with a web-server. A WatchGuard firewall will organize the communications. This platform aims at illustrating some applications oriented towards Smart Buildings and also in the field of Internet of Things.
For each of these platforms, a technical work will be achieved in Grenoble in order to set the platforms. It is aimed that the platforms will be installed, configured, they may be used for training, in Grenoble, and then the platforms will be sent in Asia.

The pedagogic aspect is also important. We have defined the contents of the platforms according to some concepts we consider to be relevant for engineering programs nowadays. The purpose of the project is to build a network of centers of excellence, in order for the Asian partners of the project, to keep working altogether and sharing their experiences and applications field altogether. As explained before, 6 centers of excellence will be set in 6 universities in South-East Asia and each center will be equipped of devices of a specific trade mark, a specific application field (ex: smart buildings, industrial processes, electrical engines), and a specific field of investigation (wireless networks, supervision, cyber-security). It is planned to develop in Grenoble the pedagogical documents for the various platforms (3) managed by Grenoble.

Once the platform installed in Grenoble and the pedagogical resources designed, a workshop will be organized in Grenoble in March 2022. This 2-week workshop will be organized for the 3 Asian partners. 4 people from each of the Asian partners will attend the workshop. The purpose of this workshop is for the colleagues to discover the platforms, work on them, to appropriate both the technical and the pedagogical aspects, in order in the future for these teachers, to be able to manage and to use these platforms for students.

As mentioned before, 6 such platforms, 3 from Ruse [1] and 3 from Grenoble, will be deployed toward the 6 Asian partners. As said before, these 6 platforms are representative of various companies and various applications. As a whole, the 6 Centers of Excellence constitute a network of centers of excellence throughout these 3 countries of South-East Asia, allowing exchanges of competences and experiences between the various members.

Finally, these platforms are designed and prepared, technically and pedagogically, in Europe. Then, the Asian partners will come in Grenoble to be taught how to use these platforms with students. Finally, the platforms will be transferred to Asia.

\section{INVOLVEMENTS OF STUDENTS}

Students are of course one of the main targets of this project. For the first phase of the project which was held in 2020, and concerns the seminars (see section III), 6 seminars were given to each of the 6 Asian partners, which means 36 seminars as a whole. Depending on the seminars and the target partners, between 30 and 100 students attended these seminars. Actually, at the time the paper is written, the seminars are not finished yet. Some evaluation questionnaires have been prepared and began to be filled back by the students. Since the set of seminars is not finished yet, the studies of the answers have not been done yet. Quizzes have also been prepared for each of the seminars with some questions about the most important concepts brought by the seminars in order to assess the main concepts have been understood and learnt by students.

In the future, presumably in the 2022-23 academic year, some student projects will be proposed, in partnership by the Asian and European teachers, for some Asian students. The 
purpose will be for the various selected Asian students to prepare and develop their study projects on the platforms developed during the project. The students will work on the platform in Asia and will be co-supervised locally by the Asian teachers and remotely by the European partners.

\section{ORganization OF A TRAINING PERIOD}

In Grenoble, we are developing the Centers of Excellences for three Asian partners: ITC Phnom Penh Cambodia, MFU Chiang Rai Thailand, and NOUL Vientiane Laos. We have now planed a Training period in Grenoble, at the end of January 2022, hoping that the pandemic will not disturb this calendar. 4 people from each of the three Asian partners will come and attend this training. This training will last two weeks. The content of these two weeks will be organized according to this preliminary content, in three sub-parts.

The third sub-part regards courses: the purpose will be to give the partners some basics or more advanced concepts regarding their centers of excellence. We may remind here that for the three Asian partners, the Centers of Excellence are organized around PLCs, from three different brands, and with three different applications: cyber-security, emulation and wireless communications. The course will be adapted to the exact background of the Asian colleagues and their expectations, and will content five sections. The first section is an introduction to PLCs: what are PLCs, how they can be used, their hardware architecture and configuration, the way to program them, with practical examples, linked to the actual hardware architecture of each PLC. The second section deals with the PLC within its environment, and supervision issues. The purpose of this section will be to present the interactions between the PLC and the supervision, the CIM architecture, the various network protocols potentially existing. Then the main issue of this class will be to present and explain what is supervision. The third section focuses on cyber-security issues: this part will be dedicated to a presentation of the main concepts and challenges around cyber-security, the specific challenges of Industrial Control Systems, and the way to test some cyber-security issues practically on the lab. The fourth section deals with emulation aspects, how to use the emulation card, how to develop emulated processes (which will be an interesting issue for some student projects in Asia) and how to interact with the emulation card within the lab. The fifth section will deal with wireless networks, what are the main challenges in the industry 4.0 environment, and how to use them within the proposed lab.

Each of the five sections described above will last around half a day. These courses will give everybody a general overview of the needed knowledge, even if each of the specific center of excellence is specialized in one of the topics, considering first that some of these aspects are general background interesting for everybody and also that depending on the needs of local students or companies, it may be interesting to evolve in the future beyond the first need expressions of the centers of excellence. This part will be interesting for the Asian partners also to prepare their own courses, for the future.

The second sub-part is the labs. This is the most important part of the training since the Asian colleagues will have work on the labs, on the specific PLCs they will have for their centers of excellence, and so this training is really oriented towards "training of trainers".

The third and last sub-part is the "social" activity. It may be interesting for the Asian colleagues to use the opportunity to be in Grenoble to visit some research labs and maybe some schools, depending on what they are interested in. We will provide them with some information in order to organize this part based on their needs and interests. Another social aspect will be the discovery of the city of Grenoble and the surroundings, possibly.

\section{OTHER ACTIONS IN THE FUTURE}

Among the other actions for the future, we may mention the setting of a Methodology for long term assessment, in order to transfer to our Asian partners, the best practices used. We can for that rely on previous works we have achieved within European projects [5].

\section{CONCLUSION}

This project is an interesting concrete project allowing to transfer the better knowledge and know-how in the field of automation in the frame of the Industry 4.0 environment. The main aspects of automation: control, instrumentation, supervision, but also communication (wired and wireless), dependability and even cyber-security in an industrial context, are studied. Each of the 6 Asian partners will have its own "center for excellence" and altogether they will constitute a "network" of centers of excellence, apprehending the various aspects of automation and equipment from various manufacturers. At this stage, and due to some delays because of the pandemic situation, we have around one year of delay, despite the fact that some actions were organized remotely. Some of the actions (technical development of the platform, training on the platforms) need to be organized face-to-face. After the processing of the questionnaires related to the seminar action this academic year, the next year will be dedicated to the centers of excellence: technical and pedagogical developments, training of Asian colleagues, transfer of the platforms to Asia.

\section{ACKNOWLEDGMENT}

This paper is based on a project co-funded by the ERASMUS+ Programme of the European Union: Asean Factori 4.0, Across South East Asian Nations: From Automation and Control Training to the Overall Roll-out of Industry 4.0, 609854-EPP-1-2019-1-FR-EPPKA2-CBHE-JP.

\section{REFERENCES}

[1] P. Daskalov, T. Georgieva, N. Bencheva, H. Yahoui. Innovative PLC training laboratory as a part of the Center of Excellence in the context of the ASEAN Factori 4.0 project. EAEEIE 21, Prague, Czech Republic.

[2] JM Thiriet, S. Mocanu. Some Considerations on Dependability Issues and Cyber-Security of CPS. SACONET'18, El Oued, Algeria.

[3] JM Thiriet, S.Mocanu. A course in cyber-security, with orientations towards cyber-physical systems. EAEEIE'19, Ruse, Bulgaria.

[4] ELLEIEC Project Lifelong learning in ELECTRICAL and INFORMATION ENGINEERING in Europe 2008-2012, 2012.

[5] W. Grega \& al. Methodology for Creation of Multidisciplinary, Transatlantic Engineering Program in Information Technology EAEEIE'09, Valencia, España. 